\title{
A Model of Applied Physiotherapy in Patellofemoral Pain \\ Konstantinos Papadopoulos*
}

Department of Science and Technology, London Sport Institute, Middlesex University, UK

\begin{abstract}
Patellofemoral Pain (PFP) is a very common knee condition with various aetiologies. This short communication facilitates a 'collective' case study approach that draws together a range of evidence from different 'cases'. These cases are defined as areas of evidence underpinning PFP management as an applied physiotherapy phenomenon. This communication reports the complexity of PFP in terms of the (i) evidence-base, (ii) the application of clear guidance, (iii) the actions of physiotherapists in the applied field context and (iv) and the fundamental dissonance rather than bridging of the evidence-base and applied practice.
\end{abstract}

A model of applied physiotherapy is presented that articulates how PFP should be viewed as a complex intervention and that a modified Promoting Action on Research Implementation (PARIHS) framework should be utilised to address the deficits of clinical practice.

Keyword: Physiotherapy; Patellofemoral pain; Clinical practice

\section{Introduction}

Patellofemoral Pain (PFP) is a well-recognised condition in athletes confounded by multifactorial causes [1]. Patellofemoral Pain typically presents as diffuse retropatellar and peripatellar localized pain in one of the two knees or in both [2]. The pain is aggravated by squatting, running, stair ascent and descent or by prolonged sitting with flexed knees [3]. Due to the complexity of the condition, there is no gold standard for assessment and treatment whilst an individual tailored approach is favoured by clinical physiotherapists [4].

A quick database search shows that in 2017 there are more than 70 published studies on PFP. However, most of the studies are individual studies which aimed to explore the associated risk factors, assessment and treatment techniques or clinical tests that are related to PFP without the identification of the issues surrounding PFP and applied physiotherapy practice.

Previous research of ours aimed to underpin the difficulties in PFP management and treatment within an applied context. This was done by considering PFP as a collective case study, presenting insight into the complexity of PFP and allowing theorising about the phenomenon [5]. The combination of approaches and different datasets in the study enabled an in-depth understanding to be developed and the articulation of a theoretical account that captured in general the issues surrounding PFP and applied physiotherapy practice based on the examination of the particular. For instance, the mixed method and the qualitative focus group studies revealed physiotherapists' perception of their role in treating PFP patients, enabled 'storytelling' and physiotherapists to describe PFP within the naturalistic settings of their own practice contexts, allowing the case to 'tell its own story' [6,7]. Relevant issues in the guidelines and literature based evidence was also revealed by an 'umbrella' review whereas additional studies identified application and reliability and utility of clinical tools such as clinical tests and outcome measures as well as approaches in practice [8-11]. The above results shows the poor bridging opportunities between practice and evidence leading to difficulties in physiotherapy practice, attempts to modify evidence but with limited durability and effectiveness. Also, there was organisational and regional 'Ambiguity' concerning the physiotherapist role in aligning practice to appropriate benchmarks for PFP.

Overall the relationship between the (i) evidence base, (ii) applied physiotherapy practice and (iii) tools and approaches were identified. Importantly, patient outcomes for people with PFP were a function of the interrelationship between these factors.

\section{Overall model of applied physiotherapy in PFP}

Findings of our studies and the further synthesis indicated that a model of applied physiotherapy emerged from the collective case study, based on the discrete results from each respective case. A final model was generated as part of the synthesis that identified the dynamics of practice environments and accounted for the influence of different variables that impacted on the outcomes of physiotherapy practice delivering interventions in PFP. The model highlighted that the implementation of a consistent and appropriate management of PFP operated on a continuum ranging from 'intra-physiotherapy practice', 'inter-physiotherapy practice' to 'extra physiotherapy -practice' (Figure 1). In this way within each component of practice a number of factors operated as mechanisms to shape and direct practice and potential outcomes for patients with PFP. A critical juncture was the 'inter-physiotherapy practice' that represented a potential gateway or barrier between the inner mechanisms of 'intra-physiotherapy practice' and the influences of environmental, regional and evidence variables in the 'extra physiotherapy - practice' end of the continuum. The extraphysiotherapy practice includes all these 'wider environmental' factors that affect PFP physiotherapy practice. As a series of critical junctures they were subject to the flow or restriction in the movement of evidence to guide PFP management with applied practice.

Within the key area of 'intra-physiotherapy practice' (Figure 1) a number of common key elements which were related to how physiotherapists assess and treat PFP patients in clinical settings were evident when questioning National Health Service (NHS) clinical physiotherapists [6,7].

Individual approach: At the core of the results was the dominance of autonomous practice by the physiotherapist, deeply influencing approaches adopted towards PFP. For instance, in terms of beliefs about causes of the syndrome, it was noted that;

${ }^{*}$ Corresponding author: Konstantinos P, Department of Science and Technology, London Sport Institute, Middlesex University, UK, Tel: 442084114372; E-mail: K.Papadopoulos@mdx.ac.uk

Received Febeuary 13, 2018; Accepted March 07, 2018; Published March 14 2018

Citation: Papadopoulos K (2018) A Model of Applied Physiotherapy in Patellofemoral Pain. Prim Health Care 8: 291. doi: 10.4172/2167-1079.1000291

Copyright: ( 2018 Papadopoulos K. This is an open-access article distributed under the terms of the Creative Commons Attribution License, which permits unrestricted use, distribution, and reproduction in any medium, provided the original author and source are credited. 


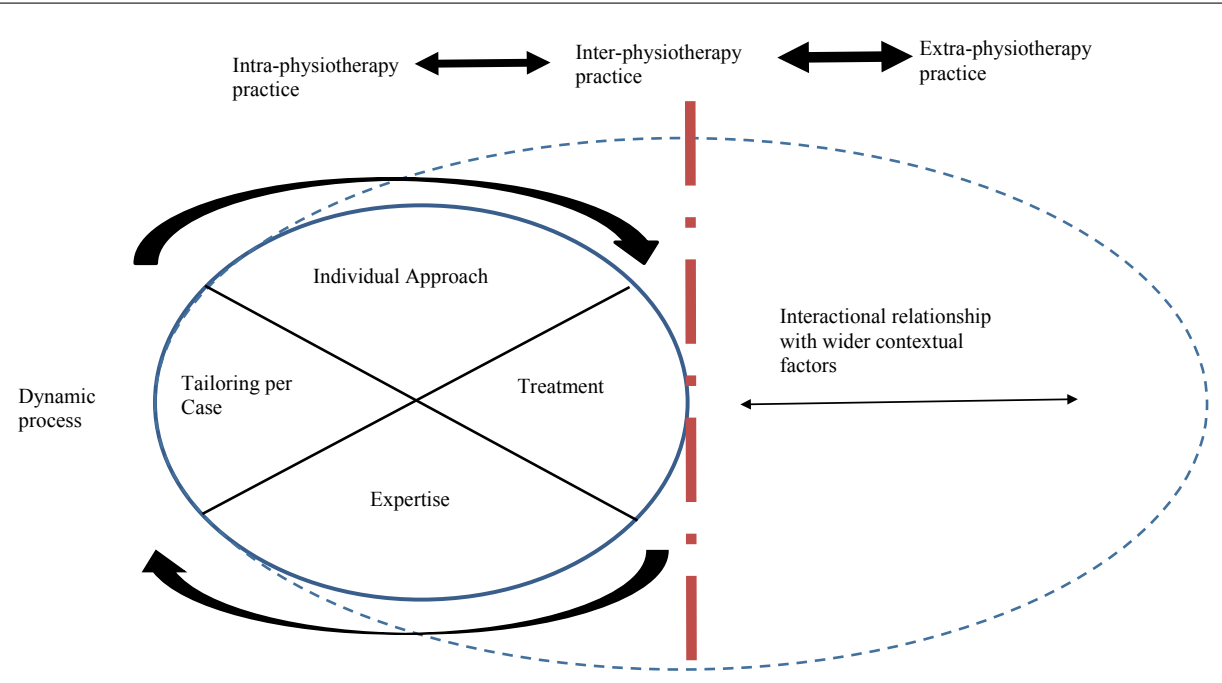

Figure 1: Applied physiotherapy practice in PFP model.

"It can be anything... It is a very challenging syndrome... any part of the leg can be the cause... bad biomechanics mostly" (Participant 4).

The complex area of PFP highlighted the significant individual approach adopted by physiotherapists in applied practice generated by the autonomous nature of their role.

Tailoring per case: Within the overarching autonomous framework of practice there were examples of consistent tailoring according to case, for instance: "From what I have seen, there is no ideal treatment, but there is an ideal treatment for each patient" (Participant 2). As such there was a poor degree of standardised practice and a significant proportion of interpretation by individual practitioners and accordingly different approaches adopted.

Expertise: Along with autonomous practice and a highly individualised approach there was the development and application of a range of expert practice to patients with PFP. In this way applied practice represented different degrees of expertise depending on the exposure to PFP and the available evidence base. For instance, home exercise programmers were utilized but only in a rather limited form: "I ask them to perform the same exercises they do here (physiotherapy department)". (Participant 6). This represented a diverse range of what could be constituted as expertise and appropriateness for patients with PFP: "It depends... If they have time, I ask them to do 5-10 exercises... if not 3-4 basic ones" (Participant 4).

Treatment: At the core of applied practice was treatment associated with assessments and outcome measurements by physiotherapists. Reflecting the other 'Intra- physiotherapy' components there was strong individualized framework adopted. For instance: "In general, the way I assess is similar to the exercises I ask them to do; like quadriceps and Vastus Medialis Obliquus (VMO) strengthening, functional tasks such as squats and stairs" (Participant 1). Such approaches varied significantly across different physiotherapists and constituted a lack of standardised practice, as illustrated by focusing on measurement:

(Participant 3) I usually check the muscle bulk, and the alignment.

(Researcher) Do you use ways to measure them?

(Participant 3) No, I do it visually

In terms of treatment priorities and options there was a significant differences in the interpretation of what represented best practice:

I often check where the patella lies on the knee. That is the first thing I do" (Participant 7).

"You have to do something about the swelling first; ... before anything else" (Participant 7).

"Quadriceps and gluts are the first muscles I check.... Their strength is very important" (Participant 2).

\section{Interactional relationship with wider contextual factors}

Yet the importantly individual (intra) physiotherapy practice was subject to wider influences, although the effectiveness of such contextual factors varied. For instance in terms of acquiring and maintaining knowledge and skills there were some opportunities to access a wider evidence-base and peer best-practice but these were often limited: "We go to many conferences... when we have the time" (Participant 9). Such external input to applied practice was matched by ad hoc opportunities for locality based intervention: "We have the internal services, every now and then.... and that helps a lot" (Participant 9). It was evident that wider influences were relevant in shaping the potential management of PFP by applied physiotherapists. In this context it focused on the two discrete areas of 'Inter-physiotherapy practice' and 'Extra physiotherapypractice' which will now be examined in greater detail [6,7].

The overall model highlighted the complexity of interactions within the wider context beyond the 'intra-physiotherapy practice'. It was evident that there was a dynamic process that centred on the common key components that influenced applied physiotherapy practice (Figure 2).

As part of what was defined in the model as the 'Inter-physiotherapy practice' there were a number of key components at an organisational level, operating as driving factors or barriers, representing organisational context and therefore shaping the nature of applied physiotherapy practice [7].

Peers: Individual physiotherapy practitioners were influenced in their practice towards PFP by interaction with other physiotherapy peers based on informal mechanisms and networks.

Guidance/Policy: Applied physiotherapists were subject to local guidelines and policy frameworks to guide their practice. However 


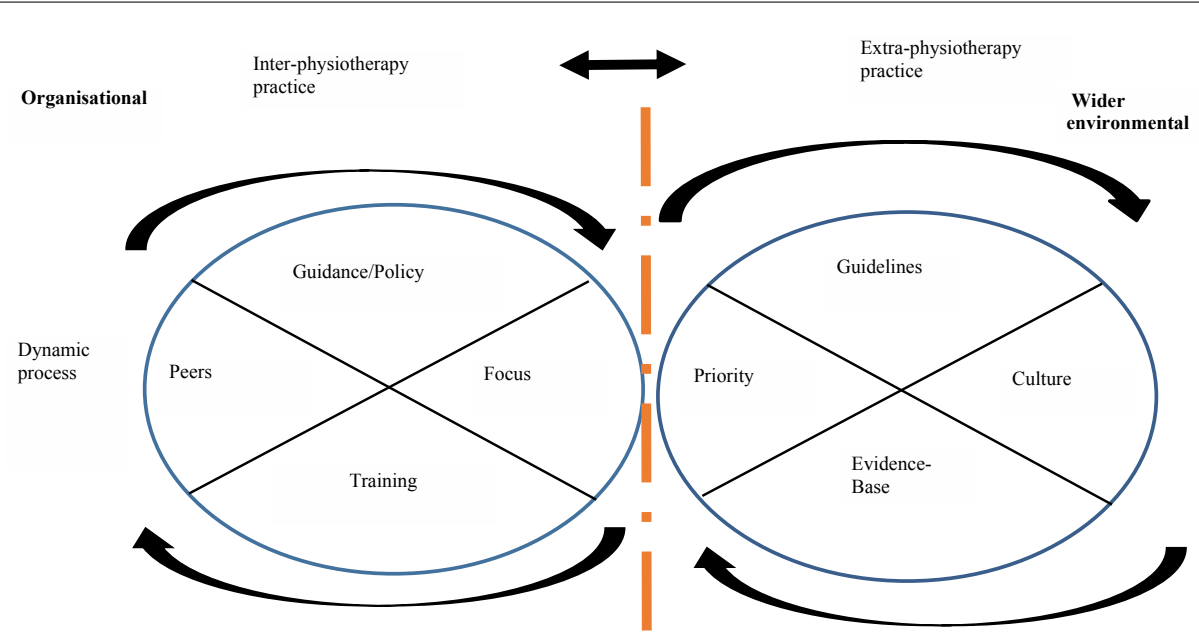

Figure 2: Critical junctures at 'intra-physiotherapy practice' and 'extra physiotherapy-practice' in model.

there was an absence of clear guidance and policy towards PFP and the role of physiotherapy was outlined only in broad terms within organisations. There was limited linkage to national or international guidelines or policy statements.

Focus: Within the applied context physiotherapists had a particular focus within their own practice which led to autonomous interventions in response to clinical presentations, framed within a broad repertoire of what constituted 'best practice'.

Training: There was generic training and development for physiotherapists but there was little in terms of supporting the role of managing PFP.

It was striking that as part of the 'Extra-physiotherapy practice' context of the model there were a further range of driving factors or barriers, which interrelated with the 'Intra-physiotherapy' and 'Interphysiotherapy' contexts [7].

Evidence-base: There existed a substantive evidence-base regarding physiotherapy practice in general terms yet there was a deficit of robust and implementation focused evidence on the management of nonathletic and athletic PFP. In this sense the nature of the evidence was problematic as there was a skewed proportion of evidence centred on athletic-management.

Priority: The degree of priority afforded to different evidence linked to physiotherapy practice was varied and within this set of priorities PFP had a generally low priority for non-athletic physiotherapy practice, with its management being ill defined and limited exposure to its presentation.

Guidelines: There was a poor framework for operationalizing best practice for PFP as guidelines were limited and implementation and dissemination to applied practice problematic.

Culture: There was a cultural context within physiotherapy practitioners of autonomy leading to a degree of not integration to guidelines and the evidence-base but separation.

\section{Implementation as a 'wicked' problem in PFP}

In this way the synthesis of the findings highlighted that PFP in applied physiotherapy practice context represented a 'wicked problem' [12]. Its main characteristic was the dissonance between physiotherapy

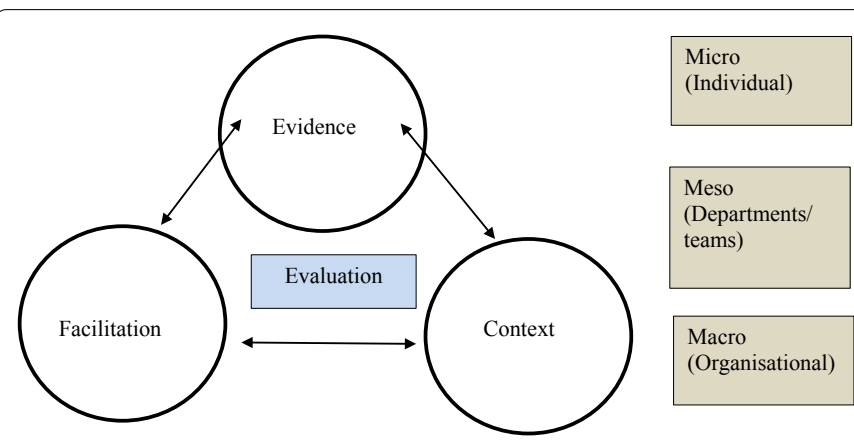

Figure 3: Modified PARIHS (Rycroft-Malone et al. [13]).

encounters with patients and the nature (as well as access to) the evidence base, including protocols and guidance. The synthesis of the findings suggested an overall model drawn from a range of variables influencing the management of PFP in applied clinical care.

\section{PARIHS and PFP}

The synthesis of the findings of our previous studies generated a model of PFP and addressed the possible opportunities for moving forward PFP treatment in applied practice. Within the PARIHS framework successful research implementation derives from the relationships among 'evidence,' 'context' and 'facilitation' [13] (Figure 3). Our Findings showed that the evidence did not inform the interventions or measurements because of the poor bridging while, the context and facilitation did not manage to inform and re-evaluate patient outcomes [7].

Rycroft-Malone et al. also suggested that successful implementation can only be defined as the use of the recommendations in practice with associated impact on practice and patient outcomes [13]. This can be achieved by incorporating the idea that there are influential factors at micro, meso and macro organizational layers of the context (in this case micro represents the individual physiotherapy practice, meso the physiotherapy departments and physiotherapy teams and macro the NHS as a complex organization (Figure 3) [13].

Based on the overall findings and the synthesis model it was evident that addressing the deficits in PFP management requires an approach that is context-centered. Across the model from 'Intra-physiotherapy' 


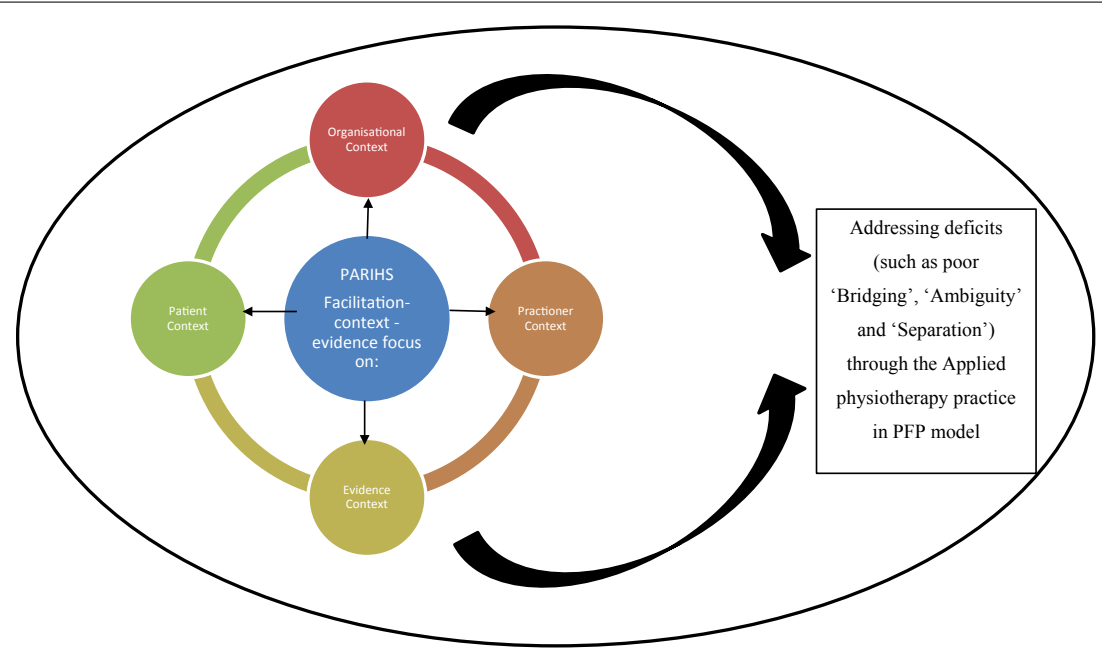

Figure 4: Dynamic development and implementation: building on the model in applied PFP' through the PARIHS framework.

to 'Extra-physiotherapy' practice the dynamic interplay of factors was shaped by differing contexts increasing barriers or operating as driving forces.

In PFP evidence-base clinical practice should be derived from a variety of sources that has been subjected to testing and has been found to be credible (Health and Care Professions Council, 2013) [14]. Within the study it would seem that physiotherapists update their knowledge with a great variety of sources however; a number of factors identified within the model highlighted the challenge for the implementation of effective knowledge-to-practice, particularly within the complex organization of the NHS context, with a range of multiple factors mitigating effectiveness. Furthermore the nature of the evidence available within the 'Wider environmental' context arguably seems located at the low-level research conducted by researchers and reported in PFP consensus statements [15]. Additionally, patient characteristics reported as part of the evidence-base seem suitable to athletic patients and not to those non-athletic patients physiotherapists see in the clinic. In this way the applied practice is restricted because of the practitioner context, the local NHS environment and wider-evidence-based factors, for instance variables linked to 'organizational' (Inter-physiotherapy) and 'wider environmental' (Extra-physiotherapy) contexts. Patient experience may be high because patients' preferences were reported to guide physiotherapy treatment (physiotherapists reported to aim at patients' problems and not at syndromes); however, this kind of approach may not show significant results on strength and flexibility.

The (Promoting Action on Research Implementation) PARIHS framework acknowledges that facilitation and facilitators play an important role in the implementation of evidence-base practice. Our studies have shown the lack of facilitation in PFP patients in NHS physiotherapy departments [6-11]. There is a need in NHS physiotherapy departments towards adapting from intra-physiotherapy practice' to 'extra physiotherapy-practice'.

The study findings suggest the utility of adopting PARIHS as a framework for addressing the deficits within current PFP practice in an applied context such as physiotherapy practice, particuarly addresing the bridging of evidence implementation into practice settings on a local level. Our studies highlighted the challenges of implementation when such a range of factors act as barriers, limiting potential drivers [6-11]. PARIHS emphasises the dynamic interrelationship between micro-meso-macro levels and the importance of evaluating contextual drivers. The final model presented in the synthesis of findings identifies and maps the relevant factors, their dynamic interrelationship and the context in relation to PFP and physiotherapy practice. PARIHS provides a framework for supporting the development of improved bridging for PFP along the 'Intra', 'Inter' and 'Extra' physiotherapy continuum with facilitation of connections between macro and micro levels by evaluation of barriers. For instance within the model there were key components that could be targeted to address improved management as part of the following based on the modelling (Figure 4).

- Practitioner Context

- Patient Context

- Evidence Context

- Organisational Context

PARIHS provides an opportunity to link the model to an appropriate implementation framework that explicitly identifies the areas that require bridging in PFP.

\section{Conclusion}

The above model mapped the analytical outcomes from the synthesis of several findings. It delineated the main features of how the applied context of physiotherapy is relevant in influencing how PFP is treated and managed. The above observations developed further the narrative that emerged from the analysis with not only a model of PFP and applied physiotherapy but also a model that identified the opportunities as well as barriers towards implementation of improvements in the treatment of PFP by physiotherapists in clinical practice. As such the PARIHS framework was highlighted as potentially providing an approach for advancing PFP based on the modelling drawn from the synthesis of our research findings.

\section{References}

1. Wood L, Muller S, Peat G (2011) The epidemiology of Patellofemoral disorder in adulthood: A review of routine general practice morbidity recording. Prim Health Care Res Dev 12: 157-164.

2. Arroll B, Ellis-Pegler E, Edwards A, Sutcliffe G (1997) Patellofemoral pain syndrome. A critical review of the clinical trials on nonoperative therapy. Am J Sports Med 25: 207-212. 
3. Gregory DM, Kevin RF, Kim DB, Arlene G, Adrick C (2010) The incidence and potential pathomechanics of patellofemoral pain in female athletes. Clin Biomech 25: 700-707.

4. Keays S, Mason M, Newcombe P (2015) Individualised physiotherapy in the treatment of patellofemoral pain. Physiother Res Int 20: 22-36.

5. Stake RE (2000) The case study method in social inquiry. In: Gomm R, Hammersley M, Foster $P$ (eds.), Case study method: Key issues. Sage Publictaions, London.

6. Papadopoulos K, Noyes J, Barnes J, Jones JG, Thom JM (2012) How do physiotherapists assess and treat patellofemoral pain syndrome in North Wales? A mixed method study. Intern J Ther Rehab 19: 261-272.

7. Papadopoulos K, Kabir R, Stasinopoulos D (2017) Perceptions of physiotherapists of their role in reducing pain and increasing function, strength and flexibility in patients with PFPS. Phy Ther Rehab 4: 1-14.

8. Papadopoulos K, Stasinopoulos D, Ganchev D (2015) A systematic review of systematic reviews of PFPS risk factors, diagnostic tests, outcome measures and exercise treatment, with an analysis of key participant and study characteristics in included primary studies. The Open Sports Med J 9: 7-17.

9. Papadopoulos K, Noyes J, Jones JG, Thom JM, Stasinopoulos, D (2014) Clinical tests for differentiating between patients with patellofemoral pain syndrome and those without. Hong Kong Physiother J 32: 35-43.
10. Papadopoulos K, Thom JM, Noyes J, Jones JG, Stasinopoulos D (2013) The reliability and meaningfulness of the anterior knee pain and lower extremity functional scales in patellofemoral pain syndrome. The Open Sports Sci J 6: 26-30.

11. Papadopoulos K, Kabir R (2016) Physiotherapy effectiveness on muscle strength, flexibility, pain and function in patients with patellofemoral pain syndrome. J Novel Physiother Phy Rehab 3: 035-039.

12. Rittel HW, Webber MM (1973) Dilemmas in a general theory of planning. Policy Sci 4: 155-169.

13. Rycroft-Malone J, Seers K, Chandler J, Hawkes CA, Crichton N, et al. (2013) The role of evidence, context, and facilitation in an implementation trial: Implications for the development of the PARIHS framework. Implement Sci 8: 8-28.

14. Health \& Care Professions Council (2013) Standards of proficiencyphysiotherapists.

15. Crossley KM, Stefanik JJ, Selfe J (2016) Patellofemoral pain consensus statement from the 4th International Patellofemoral Pain Research Retreat Manchester. Part 1: Terminology, definitions, clinical examination, natura history, patellofemoral osteoarthritis and patient-reported outcome measures. Br J Sports Med 50: 839-843. 